\title{
RON - The Con in Colorectal Carcinoma
}

\author{
Shikha Tarang and Jing Wang*
}

Eppley Institute for Research in Cancer and Allied Diseases University of Nebraska Medical Center, Omaha NE, USA

\begin{abstract}
The recepteur d'origine nantais (RON) is a member of MET family of receptor tyrosine kinase (RTKs), an overexpression of which has been observed in several cancers. The expression of RON gene is required during embryonic development and also plays critical roles in regulating macrophage inflammatory response. In CRC, the overexpression of moderate RON activity contributes to their oncogenic potential by regulating several key processes such as proliferation, motility and resistance to apoptosis. Interestingly, an aberrant RON expression is often associated with the generation of several splice variants with unique transforming activities. The targeting of RON signaling pathway by the use of monoclonal antibodies and small-molecule inhibitors has shown promising therapeutic results in animal models. The present article aims at summarizing the current understanding of RON kinase in CRC.
\end{abstract}

Keywords: Colorectal cancer, Macrophage, Receptor Tyrosine kinase, Ron kinase, Splice Variants, Therapeutic Targeting.

\section{INTRODUCTION}

Receptor Tyrosine Kinases (RTKs) are cell-surface receptors for several growth factors, cytokines and hormones [1]. Signaling by RTKs is one of the major mechanisms of intracellular communication during embryonic development and physiological processes. However during pathological conditions such as cancer either inappropriate activation or disruptions of regulatory mechanisms lead to constitutively activated pathways $[2,3]$. RON belongs to MET protooncogene family and shares significant structural and functional homology with cMET [4]. There is also a high degree of homology between MSP (Macrophage stimulating protein, the ligand for RON) and HGF/SF (Hepatocyte-growth factor/Scatter factor, the ligand for RON) [5].

RON gene is embryonic lethal in pre-implantation stage suggesting its critical role in embryonic development [6]. In adults a moderate RON gene expression is observed in several epithelial tissues and macrophages [5, 7]. The activation of RON by serum-protein MSP under physiological conditions becomes leaky in cancer consequent to several chromosomal aberrations which lead to a ligand-independent activation of RON [8]. Thus an aberrant overexpression of RON is observed in many cancers such as colon, breast and pancreatic cancers [9-13]. RON overexpression has been shown important in driving tumorigenesis and is associated with poor prognosis. Interestingly overexpression of RON is often associated with the generation of splice variants each with a unique expression profile and characteristic structural and functional properties [14-16].

Colorectal cancer (CRC) is the third most common cancer and second leading cause of cancer related deaths in the United States [17-19]. Although a number of screening

*Address correspondence to this author at the Eppley Institute for Research in Cancer and Allied Diseases University of Nebraska Medical Center Omaha, Nebraska 68198, USA; Tel: 402-559-5558; Fax: 402-559-4651;

E-mail: jjwang@unmc.edu methods have been developed, their effectiveness is limited due to lack of awareness and financial constraints $[19,20]$. Metastasis largely determines the survival time of patients and accounts for the majority of CRC deaths [21]. Upon metastasis surgical resection is limited while radiation and chemotherapy are only palliative. A molecular understanding of the phenomenon is thus urgently warranted to prolong the survival time of CRC patients with distant metastasis.

Previous studies have demonstrated the aberrant RON expression in majority of CRC patients [10, 22, 23]. Studies from our own laboratory and others have shown that RON contributes to the malignant and invasive phenotype of CRC [24]. RON has been shown to regulate several key processes in colon tumor progression and also plays active role in EMT which is analogous its role in embryonic development [25]. The function of RON in EMT is of particular clinical interest as the transition from epithelial to mesenchymal phenotype is a major determinant of metastatic dissemination [26]. Thus targeting RON is expected to have significant therapeutic efficacy in limiting the spread of metastatic CRC. Several RTK inhibitors have proven their success clinically in the past further encouraging the studies on the therapeutic utilization of RON RTK $[1,27,28]$. The characteristics of RON such as high prevalence in CRC and cell-surface RTK activity make it an important candidate for anti-cancer therapies. Further studies are warranted to develop and test the utility of targeting RON signaling in CRC. The article summarizes our present understanding on RON in pathogenesis of CRC.

\section{THE BIOLOGY OF RON}

The cloning of human RON cDNA was done less than two decades ago in 1993 from the library of human keratinocytes [29]. The frequently altered RON gene in cancers is $16.8 \mathrm{~kb}$ and located on chromosome 3p21 [29]. The mature RON receptor is $180 \mathrm{kDa}$ protein with $40 \mathrm{kDa}$ extracellular $\alpha$ - chain and $150 \mathrm{kDa} \beta$ - chain with extracellular, transmembrane and cytoplasmic domains and intrinsic kinase activity $[5,30]$. The two $\alpha$ - and $\beta$ - chains are linked by a disulphide 
bond. The extracellular domain of RON consists of well defined sema (N-terminal semaphoring), PSI (plexin semaphore-and integrin) and four immunoglobulin-like IPT domains. The only identified ligand for RON receptor is MSP (macrophage stimulating protein) [31]. The gene for MSP is located at a similar position as RON on chromosome $3 \mathrm{p} 21$ [32]. MSP is synthesized by hepatocytes and circulates in blood as inactive precursor pro-MSP [33]. The mature heterodimeric MSP consisting of a $60 \mathrm{kDa} \alpha$-chain and $30 \mathrm{kDa}$ $\beta$-chain is generated by proteolytic cleavage at dibasic arginine-valine $\left(\mathrm{R}^{554}-\mathrm{V}^{555}\right)$. The receptor-binding site of MSP is located in the MSP $\beta$-chain. The $\alpha$-chain contains four triple disulfide loop structures called kringle domains. The $\beta$-chain contains a serine protease-like domain in which there is substitution of three amino acids making it devoid of any enzymatic activities [34].

The expression of RON gene is observed early in the embryonic development. In mice homozygous loss of RON gene $\left(\operatorname{Ron}^{-1-}\right)$ leads to death prior to ED (embryonic day) 6.5 suggesting that RON is critical in embryonic development [6]. In adult tissues the expression of RON gene in epithelial cells enhances their adhesion and motility. For instance RON-MSP signaling increases the ciliary beat of nasal mucosal cells [35] and plays role in sperm motility in epithelium of the epididymis [36]. In tissue macrophages RON regulates the extent of their activation and inflammatory response [7].

\section{RON SPLICE VARIANTS}

An interesting and unique phenomenon in RON mediated tumorigenesis is the existence of splice variants. Intriguingly wild-type (wt) RON does not have cellular transforming activities on ectopic expression in NIH3T3 cells while several other variants isolated and cloned from tumor samples induce tumorigenic properties upon transformation [14, 37]. In $\mathrm{CRC}$ in addition to the overexpression of wt RON there is also existence of splice variants in certain tumor tissues. The three splice variants that have been detected are namely RON $\Delta 165$, RON $\Delta 160$, and RON $\Delta 155$ the expression and occurrence of which has been confirmed by the use of anti- bodies targeting different regions of RON [10, 23]. The splice variants have been shown to play important roles in the progression of CRC in vivo. For instance RON $\Delta 160$ and RON $\Delta 155$ when transfected in NIH3T3 cells have the ability to induce focus formation and anchorage independent growth. Further NIH3T3 cells expressing RON $\Delta 160$ or RON $\Delta 155$ form tumors in athymic nude mice and show distant metastasis to lungs [10]. The variant RON $\Delta 160$ was initially discovered in HT29 colon cancer cells [16] and later confirmed in tumor samples from CRC patients. These three RON splice variants are generated by deletions in extracellular $\beta$-domain leading to the abnormal dimerization. A comparative analysis of the different variants of RON and their oncogenic potential has been done in Table 1. Interestingly RON mutants maintain a high level of expression in CRC by resisting the process of receptor endocytosis and ubiquitylation which is observed in wt RON [38, 39].

Recently a naturally occurring antagonist of RON (RON $\triangle 85$ ) was identified in CRC and other cancers. RON $\triangle 85$ is the soluble form of RON which act by competing with wt RON for MSP binding and signaling. It is generated with an insertion of 49 nucleotides between exons 5 and 6 which introduces a premature stop codon leading to the formation of a truncated $85 \mathrm{kDa}$ RON variant consisting of entire $35 \mathrm{kDa} \alpha$-chain and a $45 \mathrm{kDa}$ partial extracellular $\beta$ chain. The protein comprises a sema domain, hinge motif and portion of the first IPT unit. Interestingly the variant RON $\Delta 85$ was not translated in cancer cells but demonstrated an inhibitory activity when used as a recombinant protein. RON $\triangle 85$ inhibits ERK1/2 and AKT phosphorylation and tumorigenic properties of CRC cells [40]. The study underscores the complexity in regulation of RON translation in cancer cells and suggests an alternative approach for RON targeted therapy.

The investigation whether a mutation in the RON kinase domain contributes to the constitutive activity in colon cancer cells was done in a panel of five carcinoma cell lines HT29, DLD-1, HCT116, SW620 and SW837. There were no mutations found but a single-nucleotide polymorphism (A4031G) in HT29 and SW620 cells [9]. Though the pres-

Table 1. The Different Splice Variants of RON and their Biological Properties in CRC

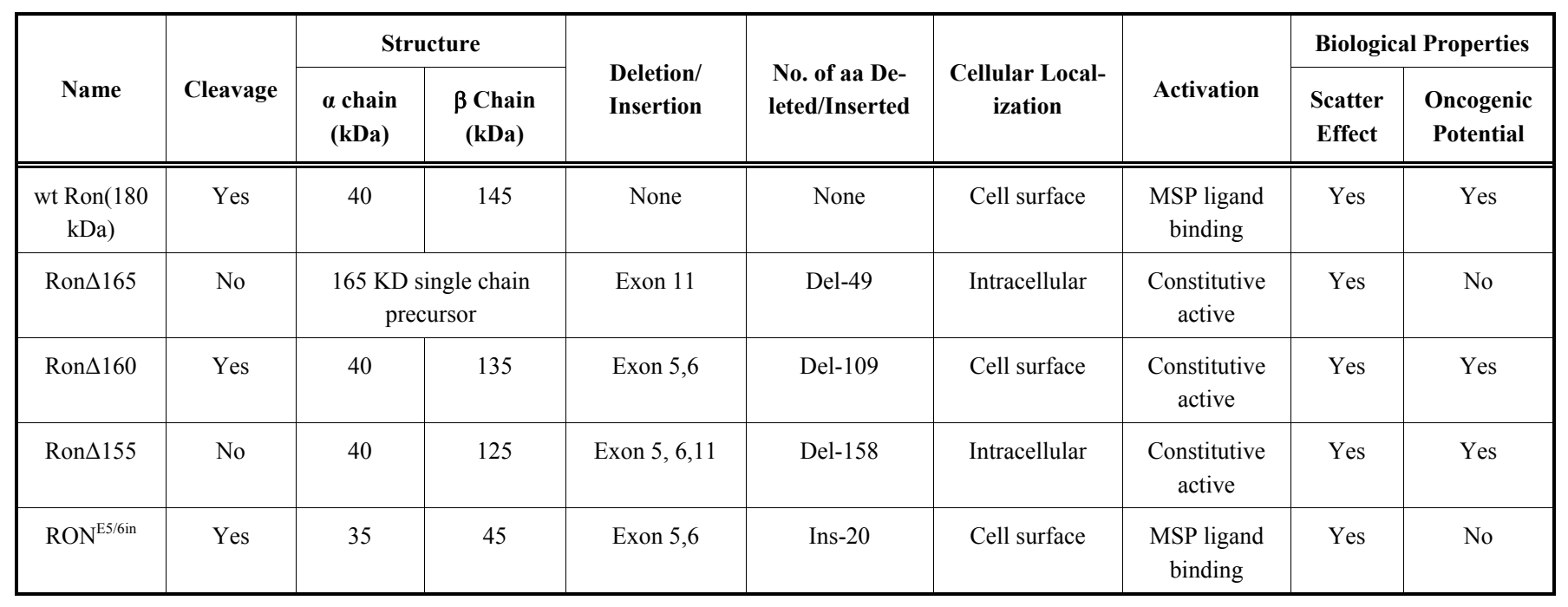


ence of mutations has not been investigated in the tumor tissues from CRC patients these initial findings indicate that receptor overexpression leading to dimerization and activation is the major mechanism of RON activity in CRC.

In order to decipher the molecular mechanisms of cancer specific RON aberrant splicing, the role of SF2/ASF splicing factor was investigated [41]. SF2/ASF on binding to the regulatory 'control cassettes' in Exon12 promotes inclusion of Exon11. Thus by regulating the ratio of wt RON and RON $\Delta 165$ transcripts SF2/ASF regulates the tumorigenic potential of the colon cancer cells. Overexpression and knockdown studies on SF2/ASF exhibited a phenotype which is similar to that of RON $\Delta 165$ further emphasizing the role of SF2/ASF in regulating its splicing [41]. In another study the role of first IPT domain in regulating the tumorigenic potential of RON was investigated in RON $\triangle 160$ and $\mathrm{RON}^{\mathrm{E} 5 / 6 \text { in }}$ which are generated by 109 amino acid deletion and 20 amino acid insertion respectively between Exon5 and Exon6 [42]. It was observed that the two variants are quite different functionally - though RON $\Delta 160$ showed constitutively active signaling, the activation of $\mathrm{RON}^{\mathrm{E} 5 / 6 \mathrm{in}}$ required stimulation with ligand MSP. Further RON $\Delta 160$ showed higher tumorigenic potential and resistance to antibody mediated receptor internalization as compared to $\mathrm{RON}^{\mathrm{E} 5 / 6 \mathrm{in}}[42]$. These findings therefore underscore the importance of IPT units in RON signaling. Further studies need to be done to comprehend fully the mechanisms of splice variants generation and a post-transcriptional regulation which translate to abnormal proteins thereby contributing to the malignant phenotype.

\section{ONCOGENIC POTENTIAL OF RON}

RON regulates several key processes in tumor development such as proliferation, motility and invasion [22, 23]. Several cancers of epithelial origin show a marked elevation in RON expression contributing to their malignant phenotype [43]. RON overexpression has high prevalence in CRC where majority of tumor tissues and cell lines from primary and metastatic CRC are shown to be positive for RON expression [43]. It is noteworthy that the inflammatory conditions such as colitis which are positively correlated with risk of CRC also show high RON expression [44].

RON was found to be over expressed and constitutively active in colon cancer cells as compared to the normal epithelial cells or SV40 transformed cells indicating the role of RON in malignant phenotype of CRC cells [9]. To elucidate whether abnormal RON activation is associated with tumorigenic properties in these cells ectopic expression of RON was carried out in SV40 transformed CoTr and colon cancer cells. It was observed that RON overexpression not only increases the migratory and invasive potential in these cells but also protects them from apoptotic stimuli [9]. The study demonstrates the biological implications of RON overexpression in pathogenesis of CRC.

The differential expression of RON in in vitro studies was corroborated by immunohistochemical (IHC) staining of the tumor tissues obtained from CRC patients [10]. In addition to the significantly higher RON staining intensity the tumor cells also showed a characteristically distinct pattern of staining as compared to the normal colon. In mucosal cells from the normal colon a granular expression of RON is observed on the cell surface and cytoplasm whereas in cancerous cells RON expression is diffused throughout the cytoplasm. The significance of the changes in expression pattern is not understood but may be indicative of their contribution to the progression of CRC [10]. An investigation into distinct staining patterns between normal and cancerous tissues might provide important clues on RON sub-cellular localization in physiological and pathological conditions. However it should be pointed here that a previous study which used another antibody for staining RON failed to identify any overexpression of RON protein in CRC [45].

In another study a comprehensive transcriptional profiling of RON and other kinases was done in different CRC stages - normal colon mucosa, adenomatous polyps, primary carcinoma and secondary metastases [46]. Though the study demonstrated a positive and differential RON expression in tumors derived from primary CRC patients it failed to detect any changes in the RON transcript in secondary colon metastasis using RT-PCR based approach [46]. Previous studies have emphasized RON is implicated in metastatic phenotype of CRC. The discrepancy in the study may be attributed to the limitation in the use of RT-PCR based approach of quantification which may not represent the actual difference in the expression pattern.

Activated RON signaling regulates the characteristic hallmarks of malignancy such as cell spreading, dissociation, migration and invasive potential $[22,47,48]$ The metastatic dissemination of epithelial cancers is often accompanied by the phenomenon of EMT which encompasses loss of epithelial properties and acquisition of mesenchymal phenotype [26]. Thus one of the main mechanisms by which RON facilitates tumor progression is by playing instrumental role in the process of EMT. RON mediated effects are manifested by associated morphological changes and in vitro assays. The biological functions of RON kinase were studied using the gene inactivation and overexpression strategies. When normal colonic cells AA/C1 were over expressed with RON they exhibited transforming phenotype of colony formation and increase in motility [49]. Similarly inactivation of RON gene significantly affected the classical tumorigenic properties of cell proliferation, migration and tumor growth in animal models of colon cancer [10,49]. These studies demonstrate that RON is critical in regulating the malignant properties of colorectal cancer. A positive RON expression in several patient tissues further emphasizes the role of RON kinase in regulating tumorigenic potential in CRC. Therefore to investigate whether RON has a role in early stage tumorigenesis, $A p c^{\mathrm{Min} /+}$ mouse was generated with and without RON signaling $\left(\mathrm{Apc}^{\mathrm{Min} /+} \mathrm{RON}^{+/+}\right.$and $\left.A p c^{\mathrm{Min} /+} \mathrm{RON}^{-/-}\right)$[50]. $A p c^{\mathrm{Min} / \mathrm{+}}$ mouse is a well studied mouse model in CRC and carries a truncation mutation at codon 850 of the $A p c$ gene and forms multiple colon tumors. The findings from these studies show that in the presence of Apc mutation RON is not required for the development and progression of CRC in mice.

\section{RON ACTIVATED SIGNALING PATHWAYS}

In physiological conditions the binding of RON with its ligand MSP causes tyrosine phosphorylation and consequent 
homo-dimerization and activation of the RON receptor. However in CRC RON receptor overexpression or generation of alternate RON splice variants lead to the ligandindependent mechanisms for RON activation $[8,51]$. It is suggested that pre-formed RON homodimers may also be activated as the result of cell interaction with the ECM [52]. The molecular mechanisms of transducing signals in the absence or presence of ligand may however be strikingly different. Notably aberrant RON activity also alters the tyrosine-phosphorylation of several proteins which may be another mechanism of RON induced cellular transformation. The activation of RON RTK regulates a number of signaling pathways such as PI3K-AKT, Ras, Src, FAK and $\beta$-Catenin which in turn regulate several key processes of malignant transformation thereby amplifying the tumorigenic potential of cells harbouring RON overexpression [53]. The role of RON was investigated in relation to deregulated mutant PI3K signaling pathway in CRC. Mutations in PIK3CA which encodes the $\mathrm{p} 110 \alpha$ catalytic subunit of PI3K are observed in a large number of CRC patients and HCT116 which has H1076R gain of function mutation. It was observed that knockdown of RON kinases inhibits the activation of mutant PI3K and thereby the metastatic potential of orthotropically injected colon cancer cells ¡24]. RON siRNA inhibited signaling by $\beta$-catenin suggesting the regulation of this pathway by RON kinase [49, 50]. However, the effect of $\mathrm{RON}$ in $\beta$-catenin expression and signaling was not observed in $A p c^{M i n /+}$ mice. The association of RON with $\beta$-catenin and PI3K signaling is highly significant with regards to pathogenesis of CRC - while $\beta$-catenin is essential for initiating the tumor formation in colon PI3K is important for cell sur- vival and also the metastatic spread and invasion at later stages of CRC progression [54-56]. Thus targeting RON RTK signaling may have profound implications in blocking several downstream and cross-activated signaling pathways Fig. (1) illustrates the RON activated signaling pathways in CRC progression.

\section{THERAPEUTIC TARGETING OF RON KINASE}

Several cancers show aberrant RTKs signaling which are the primary transducers of extracellular growth factor signals into the cell. The significance of targeting RTK signaling has been successfully demonstrated with a large number of FDA approved drugs in clinics $[1,28]$. In CRC monoclonal antibodies targeting RTKs are against VEGF (Bevacizumab) and EGFR (Cituximab and Panitumumab) [57]. An aberrant signaling from RON RTK is observed in majority of CRC patients and therefore targeting RON holds promise in achieving appropriate therapeutic outcomes. The cell-surface RON has the potential as a direct drug target or as the delivery molecule in combination with chemotherapeutic agents. The prospect of clinically targeting RON is currently being explored by the two main strategies - the development of neutralizing monoclonal antibodies and use of small molecule kinase inhibitors. The monoclonal antibodies $(\mathrm{mAb})$ used to study the effect of blocking RON signaling pathways in $\mathrm{CRC}$ include $\mathrm{Zt} / \mathrm{f} 2, \mathrm{Zt} / \mathrm{g} 4, \mathrm{Zt} / \mathrm{c} 9$ and $\mathrm{Zt} / \mathrm{c} 1$ and act by inducing receptor internalization followed by protein degradation [58-60]. Zt/g4 and Zt/c9 both recognize epitopes on the sema sequence while $\mathrm{Zt} / \mathrm{f} 2$ binds with epitopes on the IPT domain. It was observed that these mAb show a synergistic effect

\section{RON signaling IN CRC}

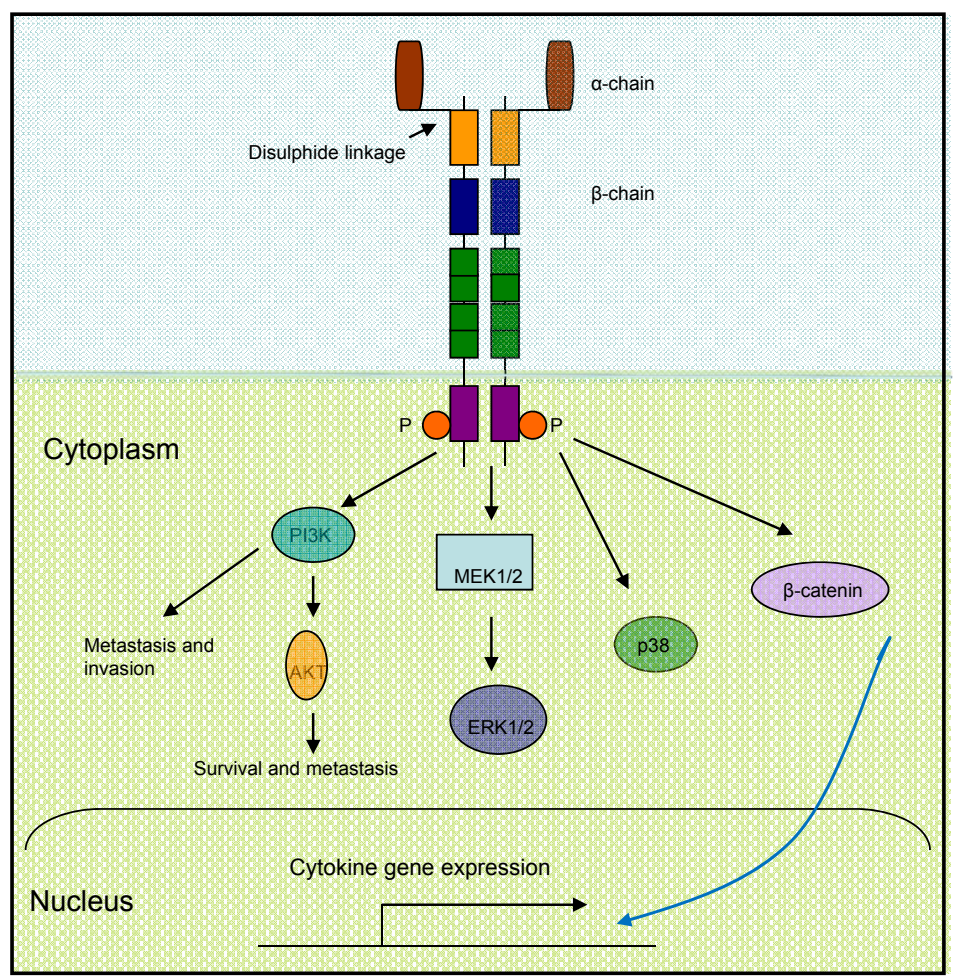

Fig. (1). The ligand-independent activation of RON leads to a constitutively active signaling pathway. The figure is representative of the know signaling pathways regulating the tumorigenic potential CRC. 
wherein a combination of any two mAbs shows a synergistic response with concomitant higher RON internalization and degradation. Further treatment with neutralizing mAbs in addition to down regulating RON- $\beta$ chain expression also inhibits the expression of pro-RON. It was observed that treatment with $\mathrm{Zt} / \mathrm{f} 2$ causes tumor regression in as early as five days of administration in xenograft mouse model [60]. An in vitro assay shows that although the antibody induces the phosphorylation of RON receptor initially it subsequently leads to receptor internalization and thus favourable biological outcomes. Further $\mathrm{Zt} / \mathrm{f} 2$ potentiates the effect of chemotherapeutic drug 5-florouracil. The tumor growth inhibitory effect is observed in both immune competent BALB/c mice and athymic nude mice deficient in T-cell mediated immunity. Treatment with $\mathrm{mAb} \mathrm{Zt/g4}$ was shown to affect the ability of colon cancer cells for colony formation in soft agar and increase the chemo-sensitivity of SW620 cells in response to gemcitabine treatment [58]. The therapeutic efficacy of blocking RON with monoclonal antibodies has also been tested in combination with other chemotherapeutic drugs. $\mathrm{Zt} / \mathrm{g} 4$ and $\mathrm{Zt} / \mathrm{cl}$ were tested for their ability to be used as a drug carrier by coating them with immunoliposome (ILP) and loading with cytotoxic drug doxorubicin (Dox) $[59,61]$. It was observed that both Anti-RON Zt/g4 and $\mathrm{Zt} / \mathrm{c} 1$ antibodies were highly effective in selectively killing colon cancer cells than pegylated liposome doxorubicin.

Further the anti-tumorigenic activities of anti-RONDOX-IL were evaluated in hypoxic cancer cells HCT116 and SW620 cells [61]. It is believed that the hypoxic conditions of the tumor microenvironment induce a drug resistance phenotype to the cancer cells [62]. Therefore the therapeutic utility of anti-RON-DOX-IL conjugate was evaluated under hypoxic conditions. It was observed that anti-RONDOX-IL treated HCT116 and SW620 cells have reduced IC50 over administration of DOX or DOX-IL. These effects were found to be dependent on hypoxic RON expression further strengthening the utility of targeting RON signaling pathway in CRC. Another RON binding antibody IMC41A10 developed by ImClone Systems has been shown to block the interaction between RON and its ligand MSP with a high affinity [63]. The efficacy of RON kinase has been demonstrated in several cancers including HT29 colon cancer cells wherein IMC41A10 has been shown to inhibit MSP induced phosphorylation and downstream signaling events. IMC41A10 is also shown to inhibit the HCA7 colon cell migration in response to MSP. Further the anti-tumor activity of IMC41A10 was shown in xenograft mouse model of HT-29 wherein it showed 50-60\% inhibition in tumor growth as a single agent [63]. Anti-RON directed receptor internalization represents an analogous situation where cells control the level of responsiveness to extracellular signals by inducing endocytosis of the receptor. Consequently therapeutic exploitation of this natural feedback mechanism to deliver cytotoxic drugs represents a promising novel approach combining inactivation of RON signaling with tumor cell killing thereby increasing the effectiveness of the therapy. The use of $\mathrm{mAb}$ in therapeutic targeting of RON suffers limitation in case of tumors where RON is activated in a ligandindependent manner as antibody presumably acts by blocking the interaction between RON and MSP [63]. Moreover certain variants of $\mathrm{RON}$ are present in the cytoplasm and consequently not amenable to antibody directed therapies. Therefore a small-molecule kinase inhibitor is expected to have broader application in RON-driven CRC. The small molecule inhibitors described so far for RON also inhibit closely related c-Met kinase. Several SMI such as quinoline based compound from Amgen, BMS-777607 from BristolMyers Squib and compound I have been described to inhibit RON/cMet kinase [64]. Compound I has been shown to result in partial inhibition of HT-29 colon xenografts [65]. However the high sequence similarity between the catalytic domains of these kinases makes the design of specific inhibitors challenging [5].

\section{RON IN TUMOR MICROENVIRONMENT}

Several studies have demonstrated that aberrant RON activity on tumor cells leads to their increased tumorigenic potential. However the role of RON in tumor microenvironment remains largely unknown. Though RON regulates the extent of activation in several populations of tissue macrophages whether it has significance in the tumor-associated macrophages (TAMs) is not well understood. The classical theory of macrophage activation proposes the existence of alternative M1 and M2 states of macrophage activation where M2 regulates the extent of inflammatory response by M1 [66, 67]. In tissue macrophages RON activation is associated with inhibiting Th-1 mediated inflammatory response to injury and inflammation. RON signaling suppresses several hallmarks of M1 polarized macrophages such as iNOS, TNF- $\alpha$ and IL-12. As RON plays active role in attenuating the inflammatory response its expression associated with M2 macrophage sub-population [68].

Tumor microenvironment is largely infiltrated by a distinct macrophages population known as tumor-associated macrophages (TAMs) which play significant roles in shaping the developing tumor. TAMs participate in carcinogenesis by facilitating and maintaining an immuno- suppressive state wherein the extent of infiltration by TAMs is positively correlated with poor prognosis in many cancers including CRC. An important metabolic switch in M1 and M2 polarization is Arg1 (arginase1) which is also associated with suppression of immune response and a tumor promoting phenotype. Recently it was shown TAMs isolated from $\mathrm{RON}^{-/}$mice show higher expression of Arg1 [68]. Further studies are warranted to decipher whether RON plays a similar role in TAMs from the CRC. If the immunosuppressive role of RON in TAMs holds true in CRC it would further strengthen the efficacy of RON targeted anti-cancer therapies.

\section{FUTURE DIRECTIONS}

The role of aberrant RON RTK signaling in pathogenesis of CRC has been established with previous studies. The existence of several RON splice variants in CRC makes it a particularly interesting molecule and needs further exploration. Though the occurrence of splice variants have been reported in CRC the functional implications of their expression in only a small subset of tumor samples remain enigmatic. Though RON signaling drives several key processes of neoplastic transformation whether colon cancer cells are addicted to RON activation is presently not known. Several kinase inhibitors such as imatinib and geftinib have been 
very successful in the clinics due to the phenomenon of oncogene addiction. Recently it is shown that cross-talk between RON and MET sustains the oncogene addiction of MET [69]. Thus study of the phenomenon of oncogene addiction with reference to RON activation in colon cancer will further strengthen the avenues for therapeutic utilization of RON RTK. In addition to the targeting of RON kinase in cancer cells, an investigation into the biological role of RON in TAMs would be highly desirable as non-malignant cells are less likely to evolve to a drug resistant phenotype.

\section{ACKNOWLEDGEMENT OF FUNDING}

The authors would like to acknowledge NIH P20RR018759 and R01CA140988-01 for the financial support.

\section{CONFLICTS OF INTEREST}

There is no conflict of interest.

\section{REFERENCES}

[1] Lemmon MA, Schlessinger J. Cell signaling by receptor tyrosine kinases. Cell 2010; 141(7): 1117-34.

[2] Kellar KA, Lorenzi MV, Ho CP, et al. Constitutively active receptor tyrosine kinases as oncogenes in preclinical models for cancer therapeutics. Mol Cancer Ther 2006; 5(6): 1571-6.

[3] Pawson T, Bernstein A. Receptor tyrosine kinases: genetic evidence for their role in Drosophila and mouse development. Trends Genet 1990; 6(11): 350-6.

[4] Dussault I, Bellon SF. From concept to reality: the long road to cMet and RON receptor tyrosine kinase inhibitors for the treatment of cancer. Anticancer Agents Med Chem 2009; 9(2): 221-9.

[5] Gaudino G, Follenzi A, Naldini L, et al. RON is a heterodimeric tyrosine kinase receptor activated by the HGF homologue MSP. EMBO J 1994; 13(15): 3524-32.

[6] Muraoka RS, Sun WY, Colbert MC, et al. The Ron/STK receptor tyrosine kinase is essential for peri-implantation development in the mouse. J Clin Invest 1999; 103(9): 1277-85.

[7] Wang MH, Zhou YQ, Chen YQ. Macrophage-stimulating protein and RON receptor tyrosine kinase: potential regulators of macrophage inflammatory activities. Scand J Immunol 2002; 56(6): 545-3.

[8] Feres KJ, Ischenko I, Hayman MJ. The RON receptor tyrosine kinase promotes MSP-independent cell spreading and survival in breast epithelial cells. Oncogene 2009; 28(2): 279-88.

[9] Chen YQ, Zhou YQ, Angeloni D, Kurtz AL, Qiang XZ, Wang $\mathrm{MH}$. Overexpression and activation of the RON receptor tyrosine kinase in a panel of human colorectal carcinoma cell lines. Exp Cell Res 2000; 261(1): 229-38.

[10] Zhou YQ, He C, Chen YQ, Wang D, Wang MH. Altered expression of the RON receptor tyrosine kinase in primary human colorectal adenocarcinomas: generation of different splicing RON variants and their oncogenic potential. Oncogene 2003; 22(2): 18697.

[11] Kretschmann KL, Eyob H, Buys SS, Welm AL. The macrophage stimulating protein/Ron pathway as a potential therapeutic target to impede multiple mechanisms involved in breast cancer progression. Curr Drug Targets 2010; 11(9): 1157-68.

[12] Maggiora P, Marchio S, Stella MC, et al. Overexpression of the RON gene in human breast carcinoma. Oncogene 1998; 16(22): 2927-33.

[13] Thomas RM, Toney K, Fenoglio-Preiser C, et al. The RON receptor tyrosine kinase mediates oncogenic phenotypes in pancreatic cancer cells and is increasingly expressed during pancreatic cancer progression. Cancer Res 2007; 67(13): 6075-82.

[14] Collesi C, Santoro MM, Gaudino G, Comoglio PM. A splicing variant of the RON transcript induces constitutive tyrosine kinase activity and an invasive phenotype. Mol Cell Biol 1996; 16(10): 5518-26.
[15] Eckerich C, Schulte A, Martens T, Zapf S, Westphal M, Lamszus $\mathrm{K}$. RON receptor tyrosine kinase in human gliomas: expression, function, and identification of a novel soluble splice variant. J Neurochem 2009; 109(4): 969-80.

[16] Wang MH, Kurtz AL, Chen Y. Identification of a novel splicing product of the RON receptor tyrosine kinase in human colorectal carcinoma cells. Carcinogenesis 2000; 21(8): 1507-12.

[17] Markowitz SD, Bertagnolli MM. Molecular origins of cancer: Molecular basis of colorectal cancer. N Engl J Med 2009; 361(25): 2449-60.

[18] Jemal A, Siegel R, Ward E, et al. Cancer statistics, 2008. CA Cancer J Clin 2008; 58(2): 71-96.

[19] Labianca R, Merelli B. Screening and diagnosis for colorectal cancer: present and future. Tumori 2010; 96(6): 889-901.

[20] Bretthauer M. Colorectal cancer screening. J Intern Med 2011; 270(2): 87-98.

[21] LeGolvan MP, Resnick M. Pathobiology of colorectal cancer hepatic metastases with an emphasis on prognostic factors. J Surg Oncol 2010; 102(8): 898-908.

[22] Camp ER, Liu W, Fan F, Yang A, Somcio R, Ellis LM. RON, a tyrosine kinase receptor involved in tumor progression and metastasis. Ann Surg Oncol 2005; 12(4): 273-81.

[23] Wang MH, Yao HP, Zhou YQ. Oncogenesis of RON receptor tyrosine kinase: a molecular target for malignant epithelial cancers. Acta Pharmacol Sin 2006; 27(6): 641-50.

[24] Wang J, Rajput A, Kan JL, et al. Knockdown of Ron kinase inhibits mutant phosphatidylinositol 3-kinase and reduces metastasis in human colon carcinoma. J Biol Chem 2009; 284(16): 10912-22.

[25] Xiangming $\mathrm{X}$, Yun Q, Guoliang $\mathrm{Z}$, Jianjiang L, Lisong $\mathrm{T}$. Mechanisms of RON-mediated epithelial-mesenchymal transition in MDCK cells through the MAPK pathway. Braz J Med Biol Res 2011; 44(7): 634-41.

[26] Bates RC, Mercurio AM. The epithelial-mesenchymal transition (EMT) and colorectal cancer progression. Cancer Biol Ther 2005; 4(4): $365-70$

[27] Zwick E, Bange J, Ullrich A. Receptor tyrosine kinase signalling as a target for cancer intervention strategies. Endocr Relat Cancer 2001; 8(3): 161-73.

[28] Zwick E, Bange J, Ullrich A. Receptor tyrosine kinases as targets for anticancer drugs. Trends Mol Med 2002; 8(1): 17-23.

[29] Ronsin C, Muscatelli F, Mattei MG, Breathnach R. A novel putative receptor protein tyrosine kinase of the met family. Oncogene 1993; 8(5): 1195-202.

[30] Iwama A, Okano K, Sudo T, Matsuda Y, Suda T. Molecular cloning of a novel receptor tyrosine kinase gene, STK, derived from enriched hematopoietic stem cells. Blood 1994; 83(11): 31609.

[31] Angeloni D, Danilkovitch-Miagkova A, Ivanov SV, et al. Gene structure of the human receptor tyrosine kinase RON and mutation analysis in lung cancer samples. Genes Chromosomes Cancer 2000; 29(2): 147-56

[32] Yoshimura T, Yuhki N, Wang MH, Skeel A, Leonard EJ. Cloning, sequencing, and expression of human macrophage stimulating protein (MSP, MST1) confirms MSP as a member of the family of kringle proteins and locates the MSP gene on chromosome 3. J Biol Chem 1993; 268(21): 15461-8.

[33] Nanney LB, Skeel A, Luan J, et al. Proteolytic cleavage and activation of pro-macrophage-stimulating protein and upregulation of its receptor in tissue injury. J Invest Dermatol 1998; 111(4): 57381.

[34] Han S, Stuart LA, Degen SJ. Characterization of the DNF15S2 locus on human chromosome 3: identification of a gene coding for four kringle domains with homology to hepatocyte growth factor. Biochemistry 1991; 30(40): 9768-80.

[35] Sakamoto O, Iwama A, Amitani R, et al. Role of macrophagestimulating protein and its receptor, RON tyrosine kinase, in ciliary motility. J Clin Invest 1997; 99(4): 701-9.

[36] Ohshiro K, Iwama A, Matsuno K, et al. Molecular cloning of rat macrophage-stimulating protein and its involvement in the male reproductive system. Biochem Biophys Res Commun 1996; 227(1): 273-80.

[37] Santoro MM, Collesi C, Grisendi S, Gaudino G, Comoglio PM. Constitutive activation of the RON gene promotes invasive growth but not transformation. Mol Cell Biol 1996; 16(12): 7072-83. 
[38] Germano S, Barberis D, Santoro MM, et al. Geldanamycins trigger a novel Ron degradative pathway, hampering oncogenic signaling. J Biol Chem 2006; 281(31): 21710-9.

[39] Penengo L, Rubin C, Yarden Y, Gaudino G. c-Cbl is a critical modulator of the Ron tyrosine kinase receptor. Oncogene 2003; 22(24): 3669-79.

[40] Ma Q, Zhang K, Yao HP, Zhou YQ, Padhye S, Wang MH. Inhibition of MSP-RON signaling pathway in cancer cells by a novel soluble form of RON comprising the entire sema sequence. Int J Oncol 2010; 36(6): 1551-61.

[41] Ghigna C, Giordano S, Shen H, et al. Cell motility is controlled by SF2/ASF through alternative splicing of the Ron protooncogene. Mol Cell 2005; 20(6): 881-90.

[42] Ma Q, Zhang K, Guin S, Zhou YQ, Wang MH. Deletion or insertion in the first immunoglobulin-plexin-transcription (IPT) domain differentially regulates expression and tumorigenic activities of RON receptor Tyrosine Kinase. Mol Cancer 2010; 9: 307.

[43] Wang MH, Lee W, Luo YL, Weis MT, Yao HP. Altered expression of the RON receptor tyrosine kinase in various epithelial cancers and its contribution to tumourigenic phenotypes in thyroid cancer cells. J Pathol 2007; 213(4): 402-11.

[44] Hirayama I, Ide M, Asao T, Kuwano H. Receptor protein tyrosine kinase Ron is highly expressed in colorectal mucosa of ulcerative colitis patients. Hepato Gastroenterology 2007; 54(78): 1672-5.

[45] Okino T, Egami H, Ohmachi H, et al. Presence of RON receptor tyrosine kinase and its splicing variant in malignant and nonmalignant human colonic mucosa. Int J Oncol 1999; 15(4): 709-14.

[46] Chen WS, Kung HJ, Yang WK, Lin W. Comparative tyrosinekinase profiles in colorectal cancers: enhanced arg expression in carcinoma as compared with adenoma and normal mucosa. Int $\mathbf{J}$ Cancer 1999; 83(5): 579-84.

[47] Leonis MA, Thobe MN, Waltz SE. Ron-receptor tyrosine kinase in tumorigenesis and metastasis. Future Oncol 2007; 3(4): 441-8.

[48] Lu Y, Yao HP, Wang MH. Multiple variants of the RON receptor tyrosine kinase: biochemical properties, tumorigenic activities, and potential drug targets. Cancer Lett 2007; 257(2): 157-64.

[49] Xu XM, Wang D, Shen Q, Chen YQ, Wang MH. RNA-mediated gene silencing of the RON receptor tyrosine kinase alters oncogenic phenotypes of human colorectal carcinoma cells. Oncogene 2004; 23(52): 8464-74.

[50] Meyer SE, Waltz SE, Goss KH. The Ron receptor tyrosine kinase is not required for adenoma formation in $\mathrm{Apc}(\mathrm{Min} /+)$ mice. Mol Carcinog 2009; 48(11): 995-1004.

[51] Wei X, Ni S, Correll PH. Uncoupling ligand-dependent and independent mechanisms for mitogen-activated protein kinase activation by the murine Ron receptor tyrosine kinase. J Biol Chem 2005; 280(42): 35098-107.

[52] Danilkovitch-Miagkova A, Angeloni D, Skeel A, Donley S, Lerman M, Leonard EJ. Integrin-mediated RON growth factor receptor phosphorylation requires tyrosine kinase activity of both the receptor and c-Src. J Biol Chem 2000; 275(20):14783-6.

[53] Danilkovitch A, Leonard EJ. Kinases involved in MSP/RON signaling. J Leukoc Biol 1999; 65(3): 345-8.
[54] Bright-Thomas RM, Hargest R. APC, beta-Catenin and hTCF-4; an unholy trinity in the genesis of colorectal cancer. Eur J Surg Oncol 2003; 29(2): 107-17.

[55] Guo XN, Rajput A, Rose R, et al. Mutant PIK3CA-bearing colon cancer cells display increased metastasis in an orthotopic model. Cancer Res 2007; 67(12): 5851-8.

[56] Rychahou PG, Jackson LN, Silva SR, Rajaraman S, Evers BM. Targeted molecular therapy of the PI3K pathway: therapeutic significance of PI3K subunit targeting in colorectal carcinoma. Ann Surg 2006; 243(6): 833-42; discussion 43-4.

[57] Tol J, Punt CJ. Monoclonal antibodies in the treatment of metastatic colorectal cancer: a review. Clin Ther 2010; 32(3): 43753

[58] Li Z, Yao H, Guin S, Padhye SS, Zhou YQ, Wang MH. Monoclonal antibody (mAb)-induced down-regulation of RON receptor tyrosine kinase diminishes tumorigenic activities of colon cancer cells. Int J Oncol 2010; 37(2): 473-82.

[59] Guin S, Yao HP, Wang MH. RON receptor tyrosine kinase as a target for delivery of chemodrugs by antibody directed pathway for cancer cell cytotoxicity. Mol Pharm 2010; 7(2): 386-97.

[60] Yao HP, Zhou YQ, Ma Q, et al. The monoclonal antibody Zt/f2 targeting RON receptor tyrosine kinase as potential therapeutics against tumor growth-mediated by colon cancer cells. Mol Cancer 2011; 10: 82 .

[61] Guin S, Ma Q, Padhye S, Zhou YQ, Yao HP, Wang MH. Targeting acute hypoxic cancer cells by doxorubicin-immunoliposomes directed by monoclonal antibodies specific to RON receptor tyrosine kinase. Cancer Chemother Pharmacol 2011; 67(5): 107383.

[62] Wilson WR, Hay MP. Targeting hypoxia in cancer therapy. Nat Rev Cancer 2011; 11(6): 393-410.

[63] O'Toole JM, Rabenau KE, Burns K, et al. Therapeutic implications of a human neutralizing antibody to the macrophage-stimulating protein receptor tyrosine kinase $(\mathrm{RON})$, a c-MET family member. Cancer Res 2006; 66(18): 9162-70.

[64] Porter J. Small molecule c-Met kinase inhibitors: a review of recent patents. Expert Opin Ther Pat 2010; 20(2): 159-77.

[65] Zhang Y, Kaplan-Lefko PJ, Rex K, et al. Identification of a novel recepteur d'origine nantais/c-met small-molecule kinase inhibitor with antitumor activity in vivo. Cancer Res 2008; 68(16): 6680-7.

[66] Sica A, Larghi P, Mancino A, et al. Macrophage polarization in tumour progression. Semin Cancer Biol 2008; 18(5): 349-55.

[67] Mantovani A, Sozzani S, Locati M, Allavena P, Sica A. Macrophage polarization: tumor-associated macrophages as a paradigm for polarized M2 mononuclear phagocytes. Trends Immunol 2002; 23(11): 549-55.

[68] Sharda DR, Yu S, Ray M, et al. Regulation of macrophage arginase expression and tumor growth by the ron receptor tyrosine kinase. $J$ Immunol 2011; 187(5): 2181-92.

[69] Benvenuti S, Lazzari L, Arnesano A, Li Chiavi G, Gentile A Comoglio PM. Ron kinase transphosphorylation sustains MET oncogene addiction. Cancer Res 2011; 71(5): 1945-55.

Received: January 17, 2012

Revised: March 28, 2012

Accepted: March 28, 2012

(C) Tarang and Wang.; Licensee Bentham Open.

This is an open access article licensed under the terms of the Creative Commons Attribution Non-Commercial License (http://creativecommons.org/licenses/ by-nc/3.0/) which permits unrestricted, non-commercial use, distribution and reproduction in any medium, provided the work is properly cited. 